\title{
Single sevoflurane exposure increases methyl-CpG island binding protein 2 phosphorylation in the hippocampus of developing mice
}

\author{
XIAO-DAN HAN, MIN LI, XIAO-GUANG ZHANG, ZHANG-GANG XUE and JING CANG \\ Department of Anesthesia, Zhongshan Hospital, Fudan University, Shanghai 200032, P.R. China
}

Received December 16, 2013; Accepted June 9, 2014

DOI: $10.3892 / \mathrm{mmr} .2014 .2751$

\begin{abstract}
Sevoflurane is an inhaled anesthetic that is widely used in clinical practice, particularly for pediatric anesthesia. Previous studies have suggested that sevoflurane may induce neurotoxicity in the brains of neonatal mice. In the present study, the possible mechanism of neurodegeneration induced by sevoflurane in the developing brain, and the possibility that memantine treatment is able to reverse this phenomenon, were investigated. On postnatal day 7 (P7) C57BL/6 mice were continuously exposed to $1.5 \%$ sevoflurane for $2 \mathrm{~h}$ following pre-injection of saline or memantine. Methyl-CpG island binding protein 2 (MeCP2), cAMP response element-binding protein (CREB) and brain-derived neurotrophic factor (BDNF) expression in the hippocampus was measured by western blotting. Exposure to $1.5 \%$ sevoflurane resulted in increased MeCP2 phosphorylation in the hippocampus, which was reversed by memantine injection. However, neither CREB phosphorylation nor BDNF expression were significantly altered by sevoflurane treatment. The current study indicated that sevoflurane causes neurotoxicity in the developing brain, and that this may be attributed to increased MeCP2 phosphorylation in the hippocampus. It was also demonstrated that this neurotoxicity can be prevented by the N-methyl-D-aspartate glutamate receptor inhibitor memantine.
\end{abstract}

\section{Introduction}

Sevoflurane is widely used in the clinic, particularly in pediatric anesthesia. However, infants brains are at a stage at which important changes are occurring, including synapse formation and axon and dendrite growth; this renders them strongly susceptible to environmental influences, such as general anesthetics. A number of studies have demonstrated that early exposure to a variety of anesthetics can cause widespread neurodegeneration in the developing brain and

Correspondence to: Dr Jing Cang, Department of Anesthesia, Building 10, Zhongshan Hospital, Fudan University, 180 Fenglin Road, Shanghai 200032, P.R. China

E-mail: cangj_jcang@163.com

Key words: sevoflurane, neurotoxicity, P7 mice, MeCP2, memantine, hippocampus persistent learning deficits $(1,2)$. Loepke et al (3) observed that isoflurane led to apoptotic neurodegeneration, and Head et al (4) elucidated the relevant signaling pathways. Satomoto et al (5) demonstrated that exposing neonatal mice to sevoflurane results in learning deficits, in addition to autism-like abnormal social behavior. These studies together demonstrate that sevoflurane is harmful to the developing brain.

Methyl- $\mathrm{CpG}$ island binding protein $2(\mathrm{MeCP} 2)$ is a transcriptional repressor and is important in neuron maturation and normal brain function (6). Studies have suggested that MeCP2 is closely associated with NMDA receptors in the brain $(7,8)$. MeCP2 is a nuclear protein that selectively binds methylated DNA, then recruits other proteins to form an inhibition complex, thereby inhibiting the expression of various genes $(9,10)$. MeCP2 gene mutations can cause the neurodevelopmental disorder Rett syndrome (11), which can lead to cognitive impairment, motor disability and repetitive stereotyped hand movements. Up to $80 \%$ of those affected with Rett syndrome experience seizures. A number of studies have hypothesized that these symptoms may be due to defects in experience-dependent synapse maturation $(12,13)$, where normal synapse connections fail to establish in the critical period. These studies have demonstrated that MeCP2 is critical in the maturation of the nervous system and the normal functioning of nerve cells.

cAMP response element-binding protein (CREB) is a nuclear regulatory factor that may regulate gene transcription following phosphorylation of serine 133, thus enhancing the expression of multiple target genes. One study indicated that CREB is the major transcription regulatory factor of brain-derived neurotrophic factor (BDNF), and thus increases BDNF expression. This is a crucial process in the survival and differentiation of neurons (14). Another study demonstrated that isoflurane anesthesia induces neuronal apoptosis by affecting the formation of neuronal synapses in the developmental stages, potentially via the BDNF-p75-RhoA signaling pathway (4).

Memantine is a derivative of amantadine, and acts on the glutamatergic system by inhibiting NMDA receptors. It is involved in neuroprotection and acts to preserve normal synaptic function. It is approved for use in moderate-to-severe Alzheimer's disease, by the FDA and the European Medicines Agency, and has been demonstrated to produce positive effects on cognition, mood and behavior $(15,16)$. 
To investigate the molecular mechanism of neurodegeneration induced by sevoflurane, neonatal mice were exposed to sevoflurane and the protein expression levels of CREB and BDNF were assessed in the developing hippocampus. Protein expression levels of MeCP2 and the effect of pre-injected memantine were also examined, in order to determine whether memantine is able to reverse the neurodegeneration.

\section{Materials and methods}

Animals and sevoflurane exposure. All animal experiments were performed using protocols approved by the institutional animal use and care committee of the Zhongshan Hospital, Fudan University (Shanghai, China). At postnatal day 7 (P7), male C57BL/6 mice (weight, 3-5 g; Shanghai Laboratory Animal Center, Shanghai, China) were randomly divided into a sevoflurane-treated group $(n=6)$ and an air-treated control group $(n=6)$ for analysis of the effects of sevoflurane on CREB phosphorylation, BDNF expression and MeCP2 phosphorylation levels. Mice were placed in a plastic container and continuously exposed to $1.5 \%$ sevoflurane (Maruishi Pharmaceutical Co., Osaka, Japan) in air, or to air alone for $2 \mathrm{~h}$, with a gas flow of $2 \mathrm{l} / \mathrm{min}$. For further experiments, male C57BL/6 mice at postnatal day 7 (P7) were randomly divided into four groups: The sevoflurane-saline group (sevo group, $n=7$ ); the air-saline group (control group, $n=6$ ); the sevoflurane-memantine group (sevo+mem group, $n=7$ ); and the air-memantine group (mem group, $n=6$ ). Mice received $1 \mathrm{mg} / \mathrm{kg}$ saline or memantine intraperitoneally prior to sevoflurane or air treatment. The mice were then placed in a plastic container and continuously exposed to $1.5 \%$ sevoflurane in air or to air alone for $2 \mathrm{~h}$, with a gas flow of $2 \mathrm{l} / \mathrm{min}$. During exposure to sevoflurane or air, the container was heated to $37^{\circ} \mathrm{C}$ with a heating pad. The concentrations of sevoflurane, oxygen and carbon dioxide in the container were monitored with a gas monitor (Datex Cardiocap II, Datex-Ohmeda, Madison, WI, USA). Following exposure to sevoflurane or air, the mice were returned to their cages. The mice were housed six per cage and maintained on a $12 \mathrm{~h}$ light/dark cycle with access to food and water ad libitum. Two hours post-exposure the mice were sacrificed by decapitation, and their hippocampi were removed.

Arterial blood gas analyses. Arterial blood samples were obtained from the left cardiac ventricle of the mice immediately after exposure to sevoflurane, and were transferred to heparinized glass capillary tubes. Blood $\mathrm{pH}$, partial pressure of carbon dioxide in $\mathrm{mmHg}\left(\mathrm{PaCO}_{2}\right)$, partial pressure of oxygen in $\mathrm{mmHg}\left(\mathrm{PaO}_{2}\right)$, lactate $(\mathrm{Lac})$, and bicarbonate $\left(\mathrm{HCO}_{3}\right)$ were analyzed immediately after blood collection using a GEM Premier 3000 analyzer (Instrumentation Laboratory, Lexington, MA, USA).

Protein extraction and western blot analysis. Resected hippocampi were placed into 1.5-ml centrifuge tubes and preserved in liquid nitrogen. All methods were conducted on ice. An NE-PER Nuclear and Cytoplasmic Extraction kit (cat no.78835; Thermo Fisher Scientific, Waltham, MA, USA) was used to extract protein samples. All steps were conducted according to the manufacturer's instructions.
Sodium dodecyl sulphate (SDS) was added to the samples prior to boiling for $10 \mathrm{~min}$ at $100^{\circ} \mathrm{C}$. Equal quantities of protein $(15 \mu \mathrm{g})$ were used to detect the expression of the proteins of interest. Samples were electrophoresed on 10 or $15 \%$ SDS polyacrylamide gel, blotted onto polyvinylidine fluoride membranes (Bio-Rad Laboratories, Hercules, CA, USA) and then incubated with the following antibodies overnight at $4^{\circ} \mathrm{C}$ : Anti-phospho-CREB (ser133), (cat no. 06-519, EMD Millipore, Billerica, MA, USA) 1:4,000 dilution in 5\% non-fat milk; anti-CREB (cat no. MAB5432, Millipore) 1:5,000 dilution in 5\% non-fat milk; anti-BDNF (cat no. AB1779SP, Millipore) 1:1,000 dilution in 5\% non-fat milk; anti-MeCP2 (cat no. 3456P, Cell Signaling Technology, Danvers, MA, USA) 1:4,000 dilution in 5\% non-fat milk; anti-phospho-MeCP2-S421 (cat no. AP3693a, Abgent Biotech, Suzhou, China) 1:2,000 dilution in 5\% non-fat milk); and anti-actin (cat no. A5441, Sigma-Aldrich, St. Louis, MO, USA) 1:10,000 dilution in 5\% non-fat milk. The following day, the blots were incubated for $1 \mathrm{~h}$ at room temperature with horseradish peroxidase-conjugated secondary goat anti-rabbit or goat anti-mouse immunoglobulin G (Kangchen, Shanghai, China), 1:5,000 dilution in 5\% non-fat milk. Immunoreactive bands were visualized using Amersham ECL Prime Western Blotting Detection kit (cat NO.RPN2232; GE Healthcare, Chalfont St. Giles, UK). The protein signals were quantified using Quantity One software and a GS-800 Calibrated Imaging Densitometer (Bio-Rad Laboratories) and normalized to a corresponding internal reference: CREB for the exression of p-CREB-S133, MeCP2 for P-MeCP2-S421 and actin for BDNF.

Statistical analysis. All data are presented as the mean \pm standard error. Data were analyzed using the unpaired Student's t-test in Origin software, version 7.5 (OriginLab, Northampton, MA, USA). $\mathrm{P}<0.05$ was considered to represent a statistically significant difference.

\section{Results}

Sevoflurane does not induce metabolic or respiratory deterioration. Blood gas analyses indicated that there was no deterioration in respiration or metabolism in the animals following a 2-h sevoflurane exposure. All parameters were tested, including $\mathrm{PH}, \mathrm{PaCO}_{2}, \mathrm{PaO}_{2}$, oxygen saturation, Lac and $\mathrm{HCO}_{3}$. No significant differences in any of the parameters were detected between the sevoflurane and the control group ( $\mathrm{P}>0.05$; Table I).

Sevoflurane does not cause changes in CREB phosphorylation and BDNF expression in the hippocampus. Western blot analyses of hippocampal CREB phosphorylation and BDNF expression levels were performed $2 \mathrm{~h}$ following exposure to sevoflurane or air. The results indicated that there were no significant differences in levels of CREB phosphorylation (Fig. 1) and BDNF (Fig. 2) expression between the sevoflurane-treated $(n=6)$ and air-treated $(n=6)$ groups.

Sevoflurane treatment increases MeCP2 phosphorylation at the serine 421 loci in the hippocampus. Western blot analyses of hippocampal MeCP2 phosphorylation in the 
Table I. Arterial blood gas analysis.

\begin{tabular}{lcc}
\hline Arterial blood gas & Control $(\mathrm{n}=6)$ & Sevoflurane $(\mathrm{n}=6)$ \\
\hline $\mathrm{pH}$ & $7.4 \pm 0.05$ & $7.38 \pm 0.04$ \\
$\mathrm{PaCO}_{2}(\mathrm{mmHg})$ & $28.4 \pm 3.0$ & $30.1 \pm 4.0$ \\
$\mathrm{PaO}_{2}(\mathrm{mmHg})$ & $91.0 \pm 6.8$ & $88.8 \pm 5.6$ \\
$\mathrm{SaO}_{2}(\%)$ & $93.6 \pm 2.6$ & $92.7 \pm 2.5$ \\
$\mathrm{Lac}^{(\mathrm{mmol} / \mathrm{l})}$ & $1.1 \pm 0.5$ & $1.2 \pm 0.4$ \\
$\mathrm{HCO}_{3}(\mathrm{mmol} / \mathrm{l})$ & $23.8 \pm 1.6$ & $24.1 \pm 1.5$ \\
\hline
\end{tabular}

Exposure to sevoflurane did not induce significant metabolic or respiratory impairment. Analysis of arterial blood gas revealed no significant differences in any of the measured parameters between the sevoflurane group and the control one (t-test, $\mathrm{P}>0.05$ ). Pa, partial pressure; Sa, saturation.
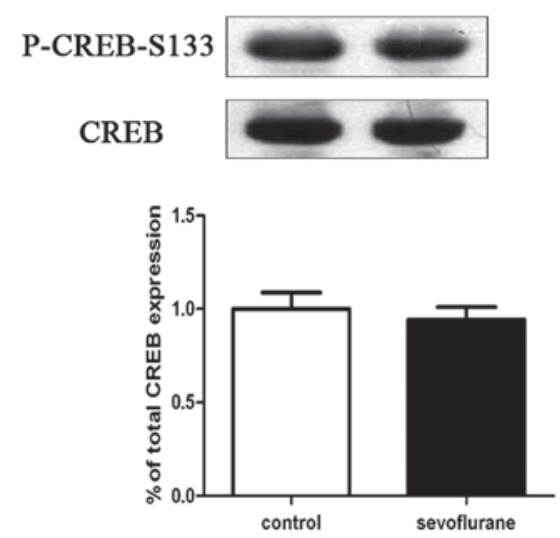

Figure 1. Sevoflurane treatment did not cause significant changes in hippocampal CREB phosphorylation in mouse hippocampi at postnatal day 7 . $\mathrm{P}>0.05$, sevoflurane group $(\mathrm{n}=6)$ vs. the control group $(\mathrm{n}=6)$. CREB, cAMP response element-binding protein.

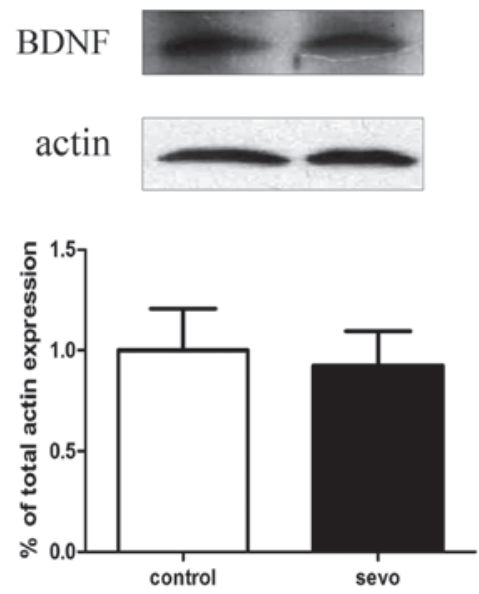

Figure 2. Sevoflurane treatment did not cause significant changes in hippocampal BDNF expression levels in mouse hippocampi at postnatal day 7. $P>0.05$, sevoflurane group $(n=6)$ vs. control group $(n=6)$. BDNF, brain-derived neurotrophic factor.

sevoflurane-treated and control groups were performed $2 \mathrm{~h}$ following exposure to sevoflurane or air. The results indicated that sevoflurane-treated mice exhibited an increase
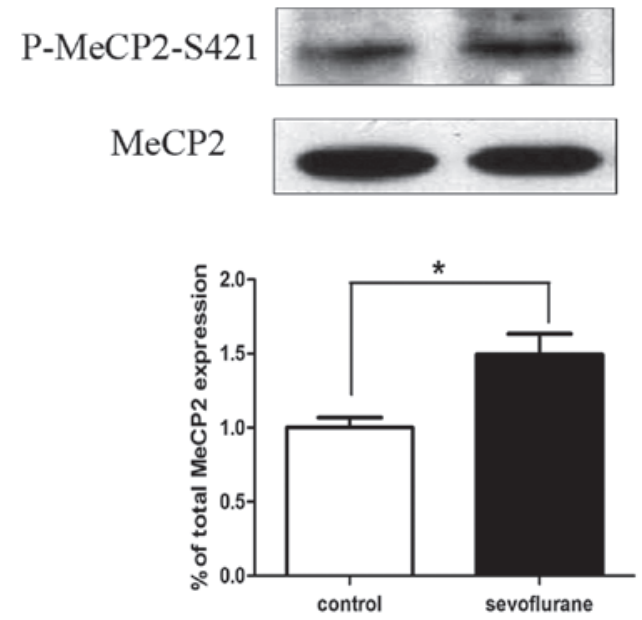

Figure 3. Sevoflurane treatment leads to a significant increase in hippocampal P-MeCP2-S421 expression levels. "P $<0.05$, sevoflurane-treated group vs. control group at $\mathrm{P} 7$. $\mathrm{MeCP} 2$, methyl- $\mathrm{CpG}$ island binding protein 2 .
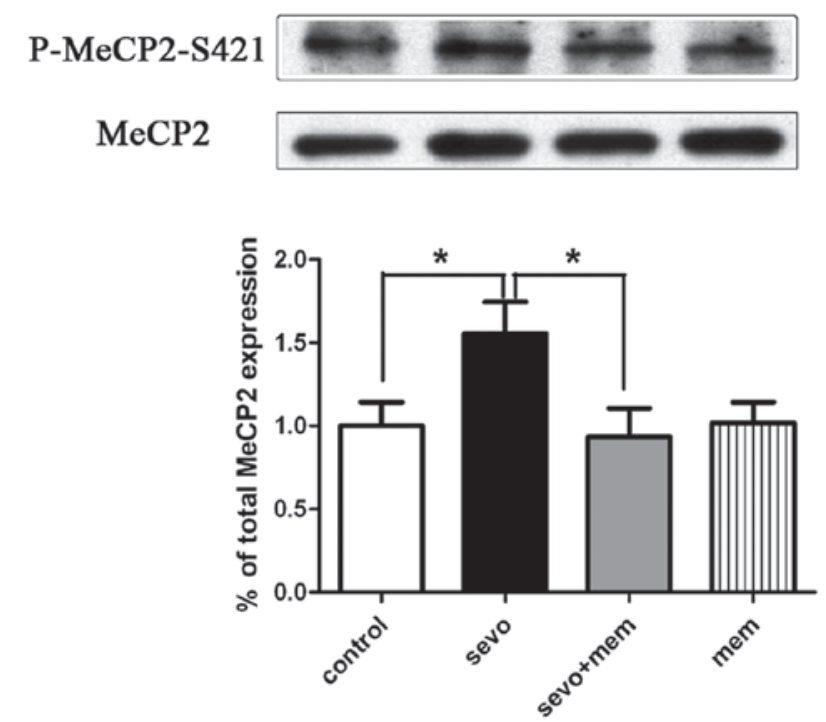

Figure 4. Memantine treatment results in normalized P-MeCP2-S421 expression levels following sevoflurane treatment. Sevoflurane-treated $(n=7)$ mice exhibited a significant increase in hippocampal P-MeCP2-S421 expression levels ( $\mathrm{P}<0.05$ vs. control group, $\mathrm{n}=6)$. The sevo + mem group $(\mathrm{n}=7)$ exhibited a significant decrease in hippocampal P-MeCP2-S421 expression levels $\left({ }^{*} \mathrm{P}<0.05\right.$ vs. sevo group). Significantly different levels of P-MeCP2-S421 expression were not identified in the mem group $(\mathrm{n}=6)$ when compared with the control group $(\mathrm{n}=6)(\mathrm{P}>0.05)$. MeCP2, methyl- $\mathrm{CpG}$ island binding protein 2.

in hippocampal $\mathrm{MeCP} 2$ phosphorylation at serine 421 loci (P-MeCP2-S421), compared with phosphorylation in the hippocampi of the control mice. There expression level of $\mathrm{P}-\mathrm{MeCP} 2-\mathrm{S} 421$ was increased in the sevoflurane-treated group $(n=6)$ compared with the control group $(n=6)(P<0.05$; Fig. 3).

Sevoflurane increases the MeCP2 phosphorylation at the serine 421 loci in the hippocampus, and pre-injection of memantine reverses this phenomenon. Western blot analyses of hippocampal MeCP2 phosphorylation were performed $2 \mathrm{~h}$ following exposure to sevoflurane or air. Compared with the control group (air and saline, $n=6$ ), the sevo group (sevoflurane 
and saline, $\mathrm{n}=7$ ) exhibited a significant increase in hippocampal P-MeCP2-S421 expression levels ( $\mathrm{P}<0.05$; Fig. 4). Compared with the sevo group, the sevo+mem group exhibited a significant decrease in hippocampal P-MeCP2-S421 levels ( $\mathrm{P}<0.05$; Fig. 4). No significant difference was detected between the mem group (memantine and saline, $\mathrm{n}=6$ ) and the control group $(\mathrm{n}=6)$.

\section{Discussion}

In the present study, it was demonstrated that P-MeCP2-S421 expression levels in hippocampi resected at $\mathrm{P} 7$ increased in mice exposed to $1.5 \%$ sevoflurane for $2 \mathrm{~h}$, and that memantine pre-injection was able to reverse this increase. However, sevoflurane did not cause significant changes in CREB phosphorylation and BDNF expression levels in the hippocampus.

A dose of $1.5 \%$ sevoflurane, which did not inhibit respiration and circulation in mouse pups, was selected for the current study. Arterial blood analyses confirmed that none of the mice experienced hypoxemia or hypercapnia during the $2-\mathrm{h}$ sevoflurane exposure; there were no significant differences in any of the tested parameters between the sevoflurane group and the control group. These results exclude the possibility of hypoxemia and hypercapnia affecting the outcome of the following experiments.

A previous study demonstrated that early exposure to sevoflurane causes widespread neurodegeneration in the developing brain (17). However, the exact mechanism of action underlying the effect of sevoflurane remains unknown. The results of the present study may provide a possible explanation for sevoflurane-mediated neurodegeneration, as they suggested that $\mathrm{MeCP} 2$ may be important in neuronal degeneration following neonatal sevoflurane exposure.

The $\gamma$-aminobutyric acid type A (GABA) and $\mathrm{N}$-methyl-D-aspartate glutamate (NMDA) receptors are essential for the development of an ordered neural map $(18,19)$, and are important in the alteration of synaptic transmission. Neurotransmitters or compounds that act on them may contribute to the impairment of brain development and synaptogenesis $(20,21)$. MeCP2 links closely with NMDA receptors in the brain, and NMDA receptors (particularly NR2A) are essential for visual cortical function in the absence of MeCP2 (22). The activity-dependent expression of another NMDA subunit (NR2B) is mediated by MeCP2-dependent epigenetic regulation (23). Thus, as indicated in the present study, $\mathrm{MeCP} 2$ may regulate NMDA receptors, leading to various effects on brain function in mice. MeCP2 phosphorylation at serine 421 loci is a key signal, which may cause downstream changes in the signaling pathway and influence the central nervous system. Previous studies indicated that CDKL5 is a target of $\mathrm{MeCP} 2$ in the brain, and is regulated by DNA methylation (24). MeCP2 can also interact with some microRNAs to regulate brain function (25). Further study of these aspects is required in order to figure out whether and how microRNAs are involved in the regulation of neuroplasticity in the brain.

Memantine is an amantadine derivative and NMDA receptor inhibitor. It has been used to treat Alzheimer's disease and is accepted to be safer than other NMDA receptor inhibitors, as a therapeutic dose of memantine has a greater affinity for synaptic NMDA receptors than extrasynaptic NMDA receptors. Thus, it exhibits an important role in neuroprotection, while preserving normal synaptic function (26). In the present study, memantine was able to reverse the increase in MeCP2 phosphorylation in the hippocampus following sevoflurane exposure. This demonstrates that memantine may have a protective effect against neurodegeneration induced by sevoflurane exposure.

In conclusion, the results of the present study demonstrated that $\mathrm{P}-\mathrm{MeCP} 2-\mathrm{S} 421$ expression levels in the hippocampus increased in P7 mice exposed to 1.5\% sevoflurane for $2 \mathrm{~h}$, and pre-injected memantine reversed this increase. Sevoflurane did not cause changes in CREB phosphorylation or BDNF expression levels in the hippocampus. Future investigation of $\mathrm{MeCP} 2$ and NMDA receptors is required in order to further investigate their effects on the central nervous system during its development.

\section{Acknowledgements}

The current study was supported by grants from the National Natural Science Foundation of China (grant no. 81100796).

\section{References}

1. Jevtovic-Todorovic V, Hartman RE, Izumi Y, et al: Early exposure to common anesthetic agents causes widespread neurodegeneration in the developing rat brain and persistent learning deficits. J Neurosci 23: 876-882, 2003.

2. Liang G, Ward C, Peng J, Zhao Y, Huang B and Wei H: Isoflurane causes greater neurodegeneration than an equivalent exposure of sevoflurane in the developing brain of neonatal mice. Anesthesiology 112: 1325-1334, 2010.

3. Loepke AW, Istaphanous GK, McAuliffe JR, et al: The effects of neonatal isoflurane exposure in mice on brain cell viability, adult behavior, learning, and memory. Anesth Analg 108: 90-104, 2009.

4. Head BP, Patel HH, Niesman IR, Drummond JC, Roth DM and Patel PM: Inhibition of p75 neurotrophin receptor attenuates isoflurane-mediated neuronal apoptosis in the neonatal central nervous system. Anesthesiology 110: 813-825, 2009.

5. Satomoto M, Satoh Y, Terui K, et al: Neonatal exposure to sevoflurane induces abnormal social behaviors and deficits in fear conditioning in mice. Anesthesiology 110: 628-637, 2009.

6. Kishi N and Macklis JD: MECP2 is progressively expressed in post-migratory neurons and is involved in neuronal maturation rather than cell fate decisions. Mol Cell Neurosci 27: 306-321, 2004.

7. Nguyen MV, Du F, Felice CA, et al: MeCP2 is critical for maintaining mature neuronal networks and global brain anatomy during late stages of postnatal brain development and in the mature adult brain. J Neurosci 32: 10021-10034, 2012.

8. Kron M, Howell CJ, Adams IT, et al: Brain activity mapping in Mecp2 mutant mice reveals functional deficits in forebrain circuits, including key nodes in the default mode network, that are reversed with ketamine treatment. J Neurosci 32: 13860-13872, 2012.

9. Cohen S, Gabel HW, Hemberg M, et al: Genome-wide activity-dependent $\mathrm{MeCP} 2$ phosphorylation regulates nervous system development and function. Neuron 72: 72-85, 2011.

10. Fuks F, Hurd PJ, Wolf D, Nan X, Bird AP and Kouzarides T: The methyl-CpG-binding protein MeCP2 links DNA methylation to histone methylation. J Biol Chem 278: 4035-4040, 2003.

11. Bienvenu T and Chelly J: Molecular genetics of Rett syndrome: when DNA methylation goes unrecognized. Nat Rev Genet 7: 415-426, 2006

12. Zhou Z, Hong EJ, Cohen S, et al: Brain-specific phosphorylation of $\mathrm{MeCP} 2$ regulates activity-dependent Bdnf transcription, dendritic growth, and spine maturation. Neuron 52: 255-269, 2006.

13. Li H, Zhong X, Chau KF, Williams EC and Chang Q: Loss of activity-induced phosphorylation of $\mathrm{MeCP} 2$ enhances synaptogenesis, LTP and spatial memory. Nat Neurosci 14: 1001-1008, 2011. 
14. Tao X, Finkbeiner S, Arnold DB, Shaywitz AJ and Greenberg ME $\mathrm{Ca}^{2+}$ influx regulates BDNF transcription by a CREB family transcription factor-dependent mechanism. Neuron 20: 709-726, 1998.

15. Areosa Sastre ASF and McShane R: Memantine for dementia. The Cochrane Library. 2006.

16. Mount $C$ and Downton C: Alzheimer disease: progress or profit? Nat Med 12: 780-784, 2006.

17. Zhang X, Xue Z and Sun A: Subclinical concentration of sevoflurane potentiates neuronal apoptosis in the developing C57BL/6 mouse brain. Neurosci Lett 447: 109-114, 2008.

18. Simon DK, Prusky GT, O'Leary DD and Constantine-Paton M: $\mathrm{N}$-methyl-D-aspartate receptor antagonists disrupt the formation of a mammalian neural map. Proc Natl Acad Sci USA 89: 10593-10597, 1992.

19. Hardingham GE, Fukunaga $\mathrm{Y}$ and Bading H: Extrasynaptic NMDARs oppose synaptic NMDARs by triggering CREB shut-off and cell death pathways. Nat Neurosci 5: 405-414, 2002.

20. Johnson SA, Young C and Olney JW: Isoflurane-induced neuroapoptosis in the developing brain of nonhypoglycemic mice. J Neurosurg Anesthesiol 20: 21-28, 2008.
21. Yon JH, Daniel-Johnson J, Carter LB and Jevtovic-Todorovic V: Anesthesia induces neuronal cell death in the developing rat brain via the intrinsic and extrinsic apoptotic pathways. Neuroscience 135: 815-827, 2005.

22. Durand S, Patrizi A, Quast KB, et al: NMDA receptor regulation prevents regression of visual cortical function in the absence of Mecp2. Neuron 76: 1078-1090, 2012.

23. Lee S, Kim W, Ham BJ, Chen W, Bear MF and Yoon BJ: Activity-dependent NR2B expression is mediated by MeCP2-dependent epigenetic regulation. Biochem Biophys Res Commun 377: 930-934, 2008.

24. Carouge D, Host L, Aunis D, Zwiller J and Anglard P: CDKL5 is a brain $\mathrm{MeCP} 2$ target gene regulated by DNA methylation. Neurobiol Dis 38: 414-424, 2010.

25. Im HI, Hollander JA, Bali P and Kenny PJ: MeCP2 controls BDNF expression and cocaine intake through homeostatic interactions with microRNA-212. Nat Neurosci 13: 1120-1127, 2010.

26. Xia P, Chen HS, Zhang D and Lipton SA: Memantine preferentially blocks extrasynaptic over synaptic NMDA receptor currents in hippocampal autapses. J Neurosci 30: 11246-11250, 2010 . 